\title{
A New Scheme for the Finite Volume Method Verified with Two Dimensional Laminar Natural Convection in a Square Cavity
}

\author{
Uthai Prasopchingchana $^{\mathrm{a}}$ and Tul Manewattana ${ }^{\mathrm{b}}$ \\ Department of Mechanical Engineering, Chulalongkorn University, Bangkok 10330, Thailand \\ E-mail: aUthai.P@Student.chula.ac.th, bTul.M@chula.ac.th (Corresponding author)
}

\begin{abstract}
A new scheme applied to the finite volume method for solving the partial differential equations of fluid flow is proposed. The Lagrange interpolating polynomial with a setting of zero for the spatial domain at the cell faces, and the present time at the cell center is adopted for the new scheme to estimate the values of the variables at the cell faces, the derivative values of the variables with respect to the spatial domain at the cell faces and the derivative values of the variables with respect to time at the cell center for spatial and temporal discretization of the discretized equation. The new scheme was verified by comparing the solutions of the new scheme to the benchmark numerical solutions and the published numerical solutions of two dimensional laminar natural convection in a square cavity. From the comparison, the results show that the solutions of the new scheme agree well with the benchmark numerical solutions and the published numerical solutions.
\end{abstract}

Keywords: Lagrange interpolating polynomial, finite volume method, laminar natural convection, square cavity.

ENGINEERING JOURNAL Volume 19 Issue 4

Received 21 November 2014

Accepted 12 January 2015

Published 31 July 2015

Online at http://www.engj.org/

DOI:10.4186/ej.2015.19.4.133 


\section{Introduction}

Computational fluid dynamics has a great deal of attention from engineering and industrial applications in the last few decades, because of saving from experimental cost and time consumption. There are many methods for numerical solving the partial differential equations of fluid flow such as the finite difference method, the finite volume method, the finite element method, the boundary element method and the lattice Boltzmann method etc. The finite volume method is one of the popular methods used to simulate the dynamic of fluids. For discretization of the equations of fluid flow, there are a lot of schemes adopted to estimate the values and derivative values of the variables of the governing equations of fluid flow. For highorder accuracy applied with non-uniform grids, this is a difficult problem for developing the discretized equations. Because a general method to determine the coefficients of schemes [1-6] is the truncated Taylor series which has to derive and calculate the coefficients of schemes from the series equations as much as the number of the accuracy order. Recently, new schemes have been proposed by using interpolating polynomial schemes [7-13]. Ratnesh et al. [7] adopted the Hemite-Birkhoff interpolation polynomials to approximate the derivative values of the variables of the high-order compact finite difference schemes for the non-uniform grids. Later, Beljadid et al. [11] used the Lagrange interpolating polynomials to estimate the values of the variables for the high-order upwind scheme. Recently, Ramirez et al. [13] employed the moving least squares interpolation polynomials to approximate the derivative values in the higher-order finite volume method to solve the incompressible Navier-Stokes equations. But all of them still have a problem with the coefficient determination for interpolation. To reduce the cumbersome process of the coefficient determination of schemes for discretization on high-order accuracy, the new scheme, the Lagrange interpolating polynomial with a setting of zero for the spatial domain at the cell faces and the present time at the cell center, is proposed in this article.

The classical verification for new schemes in the computational fluid dynamic field has been the comparison of the solutions obtained from the new schemes to the benchmark numerical solutions of natural convection in a square cavity. Natural convection in a cavity is a thermal phenomenon occurring in many engineering applications such as, air flow in gaps of double glazing units, free circulation of air in cavities of electrical equipment or electronic instrument etc. Since, the combination of fluid mechanic field and thermal science [14], verification with natural convection in a cavity can simultaneously be performed in two fields. The benchmark numerical solutions of natural convection in a square cavity with the vertical differentially heated walls and the horizontal adiabatic walls were provided by De Vahl Davis [15] and Saitoh and Hirose [16] which were obtained by using the finite difference method to discretize the equations of fluid flow. De Vahl Davis carried out the simulation with the second-order central differences for spatial discretization, while Saitoh and Hirose performed their study with the fourth-order of accuracy. In addition, nowadays there are a lot of the published numerical solutions [17-20] obtained from natural convection in a square cavity for verification of new schemes. Markatos and Pericleous [17] exhibited the solutions of laminar and turbulent natural convection in an enclosed cavity. The finite volume method was employed for simulation in their investigation. Later, Barakos et al. [18] reported the solutions of natural convection in a square cavity which they were calculated by using the finite volume method. After that, Dixit and Babu [19] presented the solutions of natural convection in a square cavity which they were obtained by using the lattice Boltmann method. Recently, Bairi [20] published the solution of natural convection in a square cavity which they were calculated by using the finite volume method.

The objective of this article is to propose the new scheme for solving the partial differential equations of fluid flow. The new scheme, Lagrange interpolating polynomial with a setting of zero of the spatial domain at the cell faces and the present time at the cell center, is employed to estimate the values of the variables at the cell faces, the derivative values of the variables with respect to the spatial domain at the cell faces and the derivative values of the variables with respect to time at the cell center of the discretized equation obtained from the transport equation of fluid flow. The fourth-order accuracy for the spatial approximation and the four time levels for temporal discretization of the new scheme are demonstrated in this article. The advantages of the new scheme are easy to determine the coefficients of the scheme and simply to extend to be the higher-order accuracy. The verification of the new scheme is based on an application, two dimensional laminar natural convection in a square cavity, while the fluid contained in the cavity is air treated as an incompressible Newtonian fluid, except the density in the body force term of the momentum equation in the vertical direction which gives the buoyancy force in the natural convection phenomenon. 


\section{New Scheme Implementation}

The finite volume method is employed to transform the transport equation of fluid flow in order to get the discretized equation and the new scheme is adopted to estimate the values of the variables at the cell faces, the derivative values of the variables with respect to the spatial domain at the cell faces and the derivative values of the variables with respect to time at the cell center for spatial and temporal discretization by using Lagrange interpolating polynomial with a setting of zero for the spatial domain at the cell faces, and the present time at the cell center.

\subsection{Lagrange Interpolating Polynomial}

The Lagrange interpolating polynomial is a powerful method for estimation. This method is able to also estimate values of the variables and derivative values of the variables with respect to the spatial domain or the temporal domain at the cell faces and the cell center of the discretized equation in the finite volume method. The general form of the Lagrange interpolating polynomial can be written as [21]

$$
F(x)=\sum_{i=1}^{n}\left(L_{i}(x) \cdot F\left(x_{i}\right)\right)
$$

where $F$ is a value of variables and a function of the spatial domain or the temporal domain $x$ and $L$ is the Lagrange interpolating polynomial coefficient. The Lagrange interpolating polynomial coefficient can be expressed as

$$
L_{i}(x)=\prod_{\substack{j=1 \\ j \neq i}}^{n} \frac{\left(x-x_{j}\right)}{\left(x_{i}-x_{j}\right)}
$$

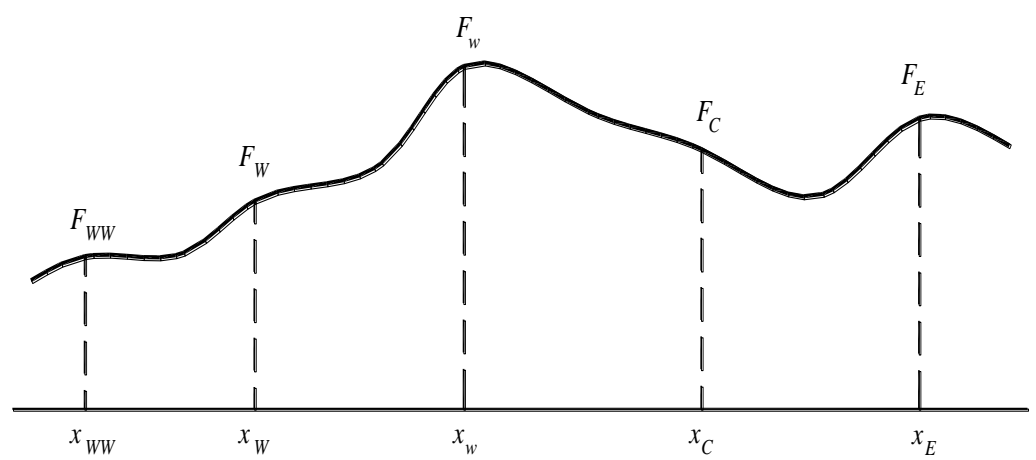

Fig. 1. Value $F$ of variables at the western cell face on the spatial domain in the $x$ direction.

For applying the Lagrange interpolating polynomial with the finite volume method for the four variable values of the neighbor cells on the spatial domain in the $x$ direction as shown in Fig. 1, when $F_{W W}, F_{W}$, $F_{C}$ and $F_{E}$ are the variable values of the neighbor cells at the spatial domain $x_{W W}, x_{W}, x_{C}$ and $x_{E}$ respectively. The value of $F_{w}$ at the western cell face can be estimated from the four variable values of the neighbor cells by using the Lagrange interpolating polynomial which it can be written as 


$$
\begin{aligned}
F_{w}= & \frac{\left(x-x_{W}\right)\left(x-x_{C}\right)\left(x-x_{E}\right)}{\left(x_{W W}-x_{W}\right)\left(x_{W W}-x_{C}\right)\left(x_{W W}-x_{E}\right)} F_{W W} \\
& +\frac{\left(x-x_{W W}\right)\left(x-x_{C}\right)\left(x-x_{E}\right)}{\left(x_{W}-x_{W W}\right)\left(x_{W}-x_{C}\right)\left(x_{W}-x_{E}\right)} F_{W} \\
& +\frac{\left(x-x_{W W}\right)\left(x-x_{W}\right)\left(x-x_{E}\right)}{\left(x_{C}-x_{W W}\right)\left(x_{C}-x_{W}\right)\left(x_{C}-x_{E}\right)} F_{C} \\
& +\frac{\left(x-x_{W W}\right)\left(x-x_{W}\right)\left(x-x_{C}\right)}{\left(x_{E}-x_{W W}\right)\left(x_{E}-x_{W}\right)\left(x_{E}-x_{C}\right)} F_{E}
\end{aligned}
$$

And the value $F_{w}$ in the Eq. (3) can be expressed in the expansion form as

$$
\begin{aligned}
F_{w}= & \frac{\left(x^{3}-\left(x_{W}+x_{C}+x_{E}\right) x^{2}+\left(x_{W} \cdot x_{C}+x_{C} \cdot x_{E}+x_{W} \cdot x_{E}\right) x-x_{W} \cdot x_{C} \cdot x_{E}\right)}{\left(x_{W W}-x_{W}\right)\left(x_{W W}-x_{C}\right)\left(x_{W W}-x_{E}\right)} F_{W W} \\
& +\frac{\left(x^{3}-\left(x_{W W}+x_{C}+x_{E}\right) x^{2}+\left(x_{W W} \cdot x_{C}+x_{C} \cdot x_{E}+x_{W W} \cdot x_{E}\right) x-x_{W W} \cdot x_{C} \cdot x_{E}\right)}{\left(x_{W}-x_{W W}\right)\left(x_{W}-x_{C}\right)\left(x_{W}-x_{E}\right)} F_{W} \\
& +\frac{\left(x^{3}-\left(x_{W W}+x_{W}+x_{E}\right) x^{2}+\left(x_{W W} \cdot x_{W}+x_{W} \cdot x_{E}+x_{W W} \cdot x_{E}\right) x-x_{W W} \cdot x_{W} \cdot x_{E}\right)}{\left(x_{C}-x_{W W}\right)\left(x_{C}-x_{W}\right)\left(x_{C}-x_{E}\right)} F_{C} \\
& +\frac{\left(x^{3}-\left(x_{W W}+x_{W}+x_{C}\right) x^{2}+\left(x_{W W} \cdot x_{W}+x_{W} \cdot x_{C}+x_{W W} \cdot x_{C}\right) x-x_{W W} \cdot x_{W} \cdot x_{C}\right)}{\left(x_{E}-x_{W W}\right)\left(x_{E}-x_{W}\right)\left(x_{E}-x_{C}\right)} F_{E}
\end{aligned}
$$

And the first order derivative of $F_{w}$ with respect to $x$ for the fourth-order accuracy is given as

$$
\begin{aligned}
\frac{d F_{w}}{d x}= & \frac{\left(3 x^{2}-2\left(x_{W}+x_{C}+x_{E}\right) x+\left(x_{W} \cdot x_{C}+x_{C} \cdot x_{E}+x_{W} \cdot x_{E}\right)\right)}{\left(x_{W W}-x_{W}\right)\left(x_{W W}-x_{C}\right)\left(x_{W W}-x_{E}\right)} F_{W W} \\
& +\frac{\left(3 x^{2}-2\left(x_{W W}+x_{C}+x_{E}\right) x+\left(x_{W W} \cdot x_{C}+x_{C} \cdot x_{E}+x_{W W} \cdot x_{E}\right)\right)}{\left(x_{W}-x_{W W}\right)\left(x_{W}-x_{C}\right)\left(x_{W}-x_{E}\right)} F_{W} \\
& +\frac{\left(3 x^{2}-2\left(x_{W W}+x_{W}+x_{E}\right) x+\left(x_{W W} \cdot x_{W}+x_{W} \cdot x_{E}+x_{W W} \cdot x_{E}\right)\right)}{\left(x_{C}-x_{W W}\right)\left(x_{C}-x_{W}\right)\left(x_{C}-x_{E}\right)} F_{C} \\
& +\frac{\left(3 x^{2}-2\left(x_{W W}+x_{W}+x_{C}\right) x+\left(x_{W W} \cdot x_{W}+x_{W} \cdot x_{C}+x_{W W} \cdot x_{C}\right)\right)}{\left(x_{E}-x_{W W}\right)\left(x_{E}-x_{W}\right)\left(x_{E}-x_{C}\right)} F_{E}
\end{aligned}
$$

When we impose that the value of $x$ equals to zero $\left(x_{w}=0\right)$, the value $F_{w}$ in Eq. (4) can be written as

$$
\begin{aligned}
F_{w}= & -\frac{x_{W} \cdot x_{C} \cdot x_{E}}{\left(x_{W W}-x_{W}\right)\left(x_{W W}-x_{C}\right)\left(x_{W W}-x_{E}\right)} F_{W W} \\
& -\frac{x_{W W} \cdot x_{C} \cdot x_{E}}{\left(x_{W}-x_{W W}\right)\left(x_{W}-x_{C}\right)\left(x_{W}-x_{E}\right)} F_{W} \\
& -\frac{x_{W W} \cdot x_{W} \cdot x_{E}}{\left(x_{C}-x_{W W}\right)\left(x_{C}-x_{W}\right)\left(x_{C}-x_{E}\right)} F_{C} \\
& -\frac{x_{W W} \cdot x_{W} \cdot x_{C}}{\left(x_{E}-x_{W W}\right)\left(x_{E}-x_{W}\right)\left(x_{E}-x_{C}\right)} F_{E}
\end{aligned}
$$

The value $F_{w}$ in Eq. (6) can be written in the short form as

$$
F_{w}=L_{W W} \cdot F_{W W}+L_{W} \cdot F_{W}+L_{C} \cdot F_{C}+L_{E} \cdot F_{E}
$$

where 


$$
\begin{aligned}
L_{W W} & =-\frac{x_{W} \cdot x_{C} \cdot x_{E}}{\left(x_{W W}-x_{W}\right)\left(x_{W W}-x_{C}\right)\left(x_{W W}-x_{E}\right)} \\
L_{W} & =-\frac{x_{W W} \cdot x_{C} \cdot x_{E}}{\left(x_{W}-x_{W W}\right)\left(x_{W}-x_{C}\right)\left(x_{W}-x_{E}\right)} \\
L_{C} & =-\frac{x_{W W} \cdot x_{W} \cdot x_{E}}{\left(x_{C}-x_{W W}\right)\left(x_{C}-x_{W}\right)\left(x_{C}-x_{E}\right)} \\
L_{E} & =-\frac{x_{W W} \cdot x_{W} \cdot x_{C}}{\left(x_{E}-x_{W W}\right)\left(x_{E}-x_{W}\right)\left(x_{E}-x_{C}\right)}
\end{aligned}
$$

In the same way, the derivative of $F_{w}$ with respect to $x$ in Eq. (5) can be written as

$$
\begin{aligned}
\frac{d F_{w}}{d x} & =\frac{x_{W} \cdot x_{C}+x_{C} \cdot x_{E}+x_{W} \cdot x_{E}}{\left(x_{W W}-x_{W}\right)\left(x_{W W}-x_{E}\right)\left(x_{W W}-x_{E E}\right)} F_{W W} \\
& +\frac{x_{W W} \cdot x_{C}+x_{C} \cdot x_{E}+x_{W W} \cdot x_{E}}{\left(x_{W}-x_{W W}\right)\left(x_{W}-x_{C}\right)\left(x_{W}-x_{E}\right)} F_{W} \\
& +\frac{x_{W W} \cdot x_{W}+x_{W} \cdot x_{E}+x_{W W} \cdot x_{E}}{\left(x_{C}-x_{W W}\right)\left(x_{C}-x_{W}\right)\left(x_{C}-x_{E}\right)} F_{C} \\
& +\frac{x_{W W} \cdot x_{W}+x_{W} \cdot x_{C}+x_{W W} \cdot x_{C}}{\left(x_{E}-x_{W W}\right)\left(x_{E}-x_{W}\right)\left(x_{E}-x_{C}\right)} F_{E}
\end{aligned}
$$

The derivative of $F_{w}$ with respect to $x$ in Eq. (8) can also be written in the short form as

$$
\frac{d F_{w}}{d x}=D_{W W} \cdot F_{W W}+D_{W} \cdot F_{W}+D_{C} \cdot F_{C}+D_{E} \cdot F_{E}
$$

where

$$
\begin{aligned}
& D_{W W}=\frac{x_{W} \cdot x_{C}+x_{C} \cdot x_{E}+x_{W} \cdot x_{E}}{\left(x_{W W}-x_{W}\right)\left(x_{W W}-x_{C}\right)\left(x_{W W}-x_{E}\right)} \\
& D_{W}=\frac{x_{W W} \cdot x_{C}+x_{C} \cdot x_{E}+x_{W W} \cdot x_{E}}{\left(x_{W}-x_{W W}\right)\left(x_{W}-x_{C}\right)\left(x_{W}-x_{E}\right)} \\
& D_{C}=\frac{x_{W W} \cdot x_{W}+x_{W} \cdot x_{E}+x_{W W} \cdot x_{E}}{\left(x_{C}-x_{W W}\right)\left(x_{C}-x_{W}\right)\left(x_{C}-x_{E}\right)} \\
& D_{E}=\frac{x_{W W} \cdot x_{W}+x_{W} \cdot x_{C}+x_{W W} \cdot x_{C}}{\left(x_{E}-x_{W W}\right)\left(x_{E}-x_{W}\right)\left(x_{E}-x_{C}\right)}
\end{aligned}
$$

The values of the variables and the derivative values of the variables with respect to the spatial domain in the $x$ and $y$ directions at the other cell faces can be estimated as this manner.

For the temporal domain as shown in Fig. $2, F_{C}$ is a value of variables at the cell center and a function of time $t . F_{C, 4}$ is a present variable value on the present time, $t_{4}$, while $F_{C, 1}, F_{C, 2}$ and $F_{C, 3}$ are the past variable values on the past time, $t_{1}, t_{2}$, and $t_{3}$. When we apply the Lagrange interpolating polynomial to the temporal function based on the implicit backward scheme with a setting of zero for present time $\left(t_{4}=0\right)$, the derivative of $F_{C}$ at the cell center with respect to time $t$ for the four time levels can be expressed as 


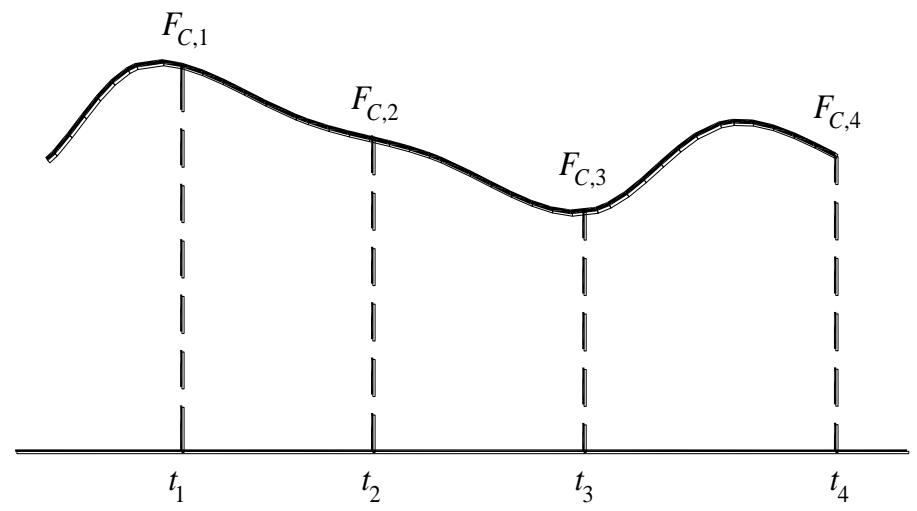

Fig. 2. Value $F_{C}$ of variables at the cell center on the temporal domain..

$$
\frac{d F_{C}}{d t}=D_{1} \cdot F_{C, 1}+D_{2} \cdot F_{C, 2}+D_{3} \cdot F_{C, 3}+D_{4} \cdot F_{C, 4}
$$

where

$$
\begin{aligned}
& L t_{1}=t_{1}-t_{4} \\
& L t_{2}=t_{2}-t_{4} \\
& L t_{3}=t_{3}-t_{4} \\
& L t_{4}=t_{4}-t_{4}
\end{aligned}
$$

and

$$
\begin{aligned}
& D_{1}=\frac{L t_{2} \cdot L t_{3}+L t_{3} \cdot L t_{4}+L t_{4} \cdot L t_{2}}{\left(L t_{1}-L t_{2}\right)\left(L t_{1}-L t_{3}\right)\left(L t_{1}-L t_{4}\right)} \\
& D_{2}=\frac{L t_{3} \cdot L t_{4}+L t_{4} \cdot L t_{1}+L t_{1} \cdot L t_{3}}{\left(L t_{2}-L t_{3}\right)\left(L t_{2}-L t_{4}\right)\left(L t_{2}-L t_{1}\right)} \\
& D_{3}=\frac{L t_{4} \cdot L t_{1}+L t_{1} \cdot L t_{2}+L t_{2} \cdot L t_{4}}{\left(L t_{3}-L t_{4}\right)\left(L t_{3}-L t_{1}\right)\left(L t_{3}-L t_{2}\right)} \\
& D_{4}=\frac{L t_{1} \cdot L t_{2}+L t_{2} \cdot L t_{3}+L t_{3} \cdot L t_{1}}{\left(L t_{4}-L t_{1}\right)\left(L t_{4}-L t_{2}\right)\left(L t_{4}-L t_{3}\right)}
\end{aligned}
$$

\subsection{Temporal and Spatial Discretization in the Finite Volume Method}

First, the partial differential equations defining fluid flow are rewritten to be a transport equation. Later, the finite volume method is employed to transform the transport equation of fluid flow into a discretized equation. The flow is assumed to be incompressible except the density in the body force term in the vertical direction, which is a function of the temperature and pressure of fluid flow. The radiation effect is excluded. The non-uniform staggered grids [22] are used for discretization. 


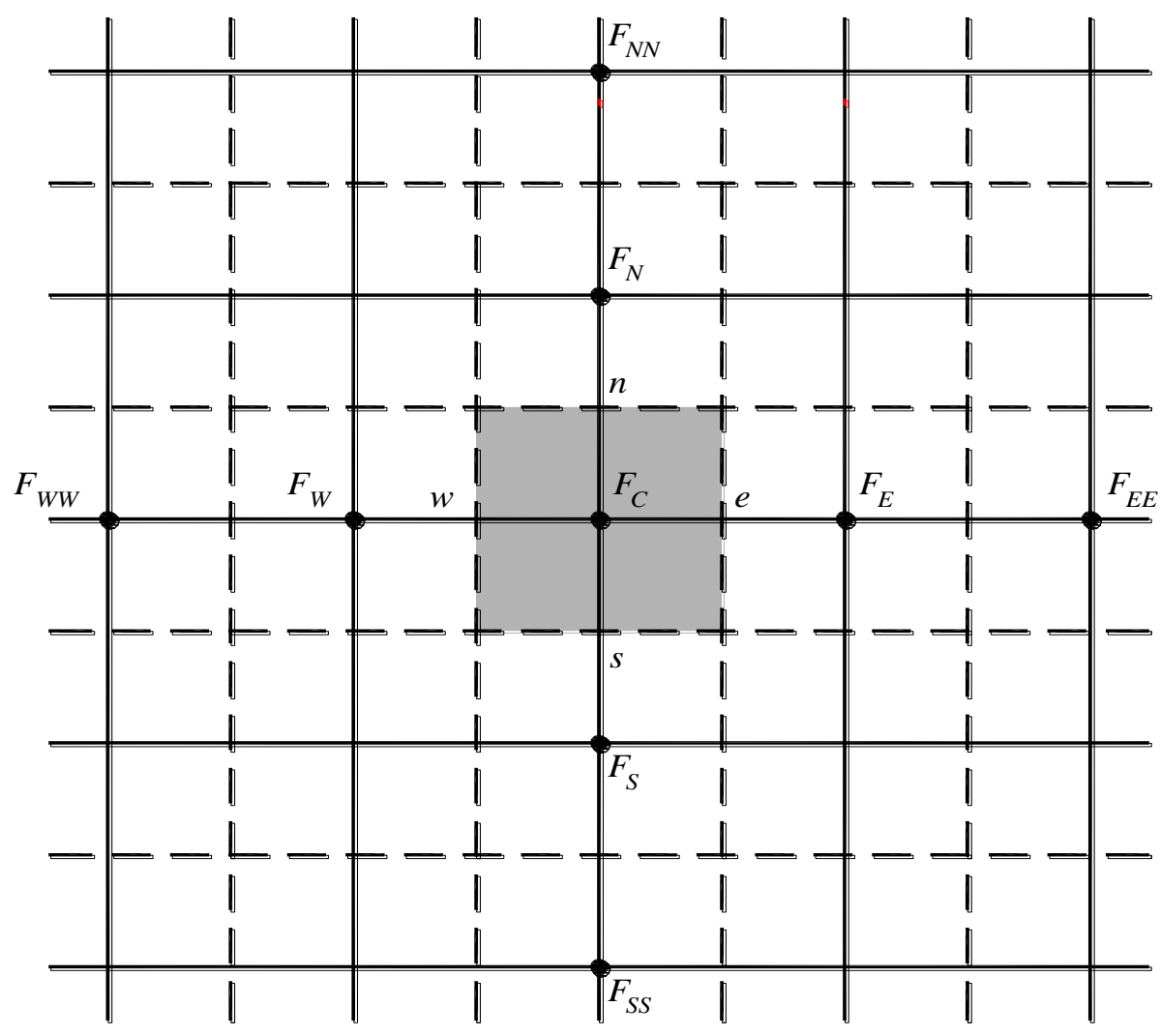

Fig. 3. Staggered grids with the values of the variables at the cell center and the neighbor cells.

The governing equations for defining two dimensional unsteady incompressible laminar viscous flow of the fluid are given by [23].

- Continuity equation

$$
\frac{\partial u}{\partial x}+\frac{\partial v}{\partial y}=0
$$

- Momentum equation in the horizontal direction

$$
\rho\left(\frac{\partial u}{\partial t}+u \frac{\partial u}{\partial x}+v \frac{\partial u}{\partial y}\right)=-\frac{\partial P}{\partial x}+\mu\left(\frac{\partial^{2} u}{\partial x^{2}}+\frac{\partial^{2} u}{\partial y^{2}}\right)
$$

- Momentum equation in the vertical direction

$$
\rho\left(\frac{\partial v}{\partial t}+u \frac{\partial v}{\partial x}+v \frac{\partial v}{\partial y}\right)=-\frac{\partial P}{\partial y}+\mu\left(\frac{\partial^{2} v}{\partial x^{2}}+\frac{\partial^{2} v}{\partial y^{2}}\right)-\rho g
$$

- Energy equation

$$
\rho c_{p}\left(\frac{\partial T}{\partial t}+u \frac{\partial T}{\partial x}+v \frac{\partial T}{\partial y}\right)=k\left(\frac{\partial^{2} T}{\partial x^{2}}+\frac{\partial^{2} T}{\partial y^{2}}\right)+\mu\left\{2\left[\left(\frac{\partial u}{\partial x}\right)^{2}+\left(\frac{\partial v}{\partial y}\right)^{2}\right]+\left(\frac{\partial u}{\partial y}+\frac{\partial v}{\partial x}\right)^{2}\right\}
$$

where $u$ and $v$ are the velocity components in the horizontal and vertical directions respectively, $x$ and $y$ are the Cartesian coordinate in the horizontal and vertical directions of the spatial domain respectively, $\rho$ is the fluid density, $P$ is the fluid pressure, $\mu$ is the viscosity of the fluid, $g$ is the gravitational acceleration (= $\left.9.81 \mathrm{~m} / \mathrm{s}^{2}\right), c_{p}$ is the specific heat value at the constant pressure of the fluid, $T$ is the fluid temperature and $k$ is the thermal conductivity of the fluid. The Eq. (12), Eq. (13) and Eq. (14) can be rewritten in a common form called the transport equation and it is expressed as

$$
\alpha \frac{\partial \phi}{\partial t}+\alpha(V \cdot \operatorname{grad}(\phi))=\Gamma \operatorname{div}(\operatorname{grad}(\phi))+S_{\phi}
$$

where 
- Momentum equation in the horizontal direction

- Momentum equation in the vertical direction

$$
\alpha=\rho, \phi=u, \Gamma=\mu, S_{\phi}=-\frac{\partial P}{\partial x}
$$

$$
\alpha=\rho, \phi=v, \Gamma=\mu, S_{\phi}=-\frac{\partial P}{\partial y}-\rho g
$$

- Energy equation

$$
\alpha=\rho c_{p}, \phi=T, \Gamma=k, S_{\phi}=\mu\left\{2\left[\left(\frac{\partial u}{\partial x}\right)^{2}+\left(\frac{\partial v}{\partial y}\right)^{2}\right]+\left(\frac{\partial u}{\partial y}+\frac{\partial v}{\partial x}\right)^{2}\right\}
$$

and $V$ is the total velocity. The finite volume method is applied to the transport equation Eq. (15) by integrating

$$
\alpha \int_{C V} \frac{\partial \phi}{\partial t} d \forall+\alpha \int_{C V}(V \cdot \operatorname{grad}(\phi)) d \forall=\Gamma \int_{C V} \operatorname{div}(\operatorname{grad}(\phi)) d \forall+\int_{C V} S_{\phi} d \forall
$$

where $C V$ is the control volume of the cells, $\forall$ is the volume of the cells, grad is the gradient of scalar variables, and div is the divergence of vector variables. When the Gauss's divergence theorem is applied to Eq. (16), it can be written as

$$
\alpha \int_{C V} \frac{\partial \phi}{\partial t} d \forall+\alpha \int_{A} n \cdot(\phi V) d A=\Gamma \int_{A} n \cdot(\operatorname{grad}(\phi)) d A+\int_{C V} S_{\phi} d \forall
$$

where $A$ is the area of the cell surfaces and $n$ is the unit normal vector of the cell faces. The Eq. (17) is discretized and expressed as

$$
\begin{aligned}
& \alpha \frac{d \phi}{d t} d x_{C} d y_{C}+\left[\left(\alpha u(\phi) d y_{C}\right)_{e}-\left(\alpha u(\phi) d y_{C}\right)_{w}+\left(\alpha v(\phi) d x_{C}\right)_{n}-\left(\alpha v(\phi) d x_{C}\right)_{s}\right] \\
& =\left[\left(\Gamma d y_{C} \frac{d \phi}{d x}\right)_{e}-\left(\Gamma d y_{C} \frac{d \phi}{d x}\right)_{w}+\left(\Gamma d x_{C} \frac{d \phi}{d y}\right)_{n}-\left(\Gamma d x_{C} \frac{d \phi}{d y}\right)_{s}\right]+S_{\phi} d x_{C} d y_{C}
\end{aligned}
$$

The Eq. (18) can be rearranged and given as

$$
\begin{aligned}
& \alpha \frac{d \phi}{d t} d x_{C} d y_{C}+\left[\left(\alpha u(\phi) d y_{C}\right)_{e}-\left(\alpha u(\phi) d y_{C}\right)_{w}+\left(\alpha v(\phi) d x_{C}\right)_{n}-\left(\alpha v(\phi) d x_{C}\right)_{s}\right] \\
& +\left[\left(\Gamma d y_{C} \frac{d \phi}{d x}\right)_{w}-\left(\Gamma d y_{C} \frac{d \phi}{d x}\right)_{e}+\left(\Gamma d x_{C} \frac{d \phi}{d y}\right)_{s}-\left(\Gamma d x_{C} \frac{d \phi}{d y}\right)_{n}\right] \\
& =S_{\phi} d x_{C} d y_{C}
\end{aligned}
$$

When the Lagrange interpolating polynomial is applied to estimate the values and derivative values with respect to the spatial domain or the temporal domain of the variables at the cell faces and the cell center of Eq. (19), it is expressed as 


$$
\begin{aligned}
& \alpha\left(D_{1} \cdot \phi_{C, 1}+D_{2} \cdot \phi_{C, 2}+D_{3} \cdot \phi_{C, 3}+D_{4} \cdot \phi_{C}\right) d x_{C} d y_{C} \\
& +\left(\alpha u\left(L_{W} \cdot \phi_{W}+L_{C} \cdot \phi_{C}+L_{E} \cdot \phi_{E}+L_{E E} \cdot \phi_{E E}\right) d y_{C}\right)_{e} \\
& -\left(\alpha u\left(L_{W W} \cdot \phi_{W W}+L_{W} \cdot \phi_{W}+L_{C} \cdot \phi_{C}+L_{E} \cdot \phi_{E}\right) d y_{C}\right)_{w} \\
& +\left(\alpha v\left(L_{S} \cdot \phi_{S}+L_{C} \cdot \phi_{C}+L_{N} \cdot \phi_{N}+L_{N N} \cdot \phi_{N N}\right) d x_{C}\right)_{n} \\
& -\left(\alpha v\left(L_{S S} \cdot \phi_{S S}+L_{S} \cdot \phi_{S}+L_{C} \cdot \phi_{C}+L_{N} \cdot \phi_{N}\right) d x_{C}\right)_{s} \\
& +\left(\Gamma d y_{C}\left(D_{W W} \cdot \phi_{W W}+D_{W} \cdot \phi_{W}+D_{C} \cdot \phi_{C}+D_{E} \cdot \phi_{E}\right)\right)_{w} \\
& -\left(\Gamma d y_{C}\left(D_{W} \cdot \phi_{W}+D_{C} \cdot \phi_{C}+D_{E} \cdot \phi_{E}+D_{E E} \cdot \phi_{E E}\right)\right)_{e} \\
& +\left(\Gamma d x_{C}\left(D_{S S} \cdot \phi_{S S}+D_{S} \cdot \phi_{S}+D_{C} \cdot \phi_{C}+D_{N} \cdot \phi_{N}\right)\right)_{s} \\
& -\left(\Gamma d x_{C}\left(D_{S} \cdot \phi_{S}+D_{C} \cdot \phi_{C}+D_{N} \cdot \phi_{N}+D_{N N} \cdot \phi_{N N}\right)\right)_{n} \\
& =S_{\phi} d x_{C} d y_{C}
\end{aligned}
$$

The Eq. (20) can be rearranged and given as

$$
\begin{aligned}
& \left(-\alpha v_{s} d x_{C} L_{S S_{-} s}+\Gamma d x_{C} D_{S S_{-} s}\right) \phi_{S S} \\
& +\left(\alpha d x_{C}\left(v_{n} L_{S_{-} n}-v_{s} L_{S_{-} s}\right)+\Gamma d x_{C}\left(D_{S_{-} s}-D_{S_{-} n}\right)\right) \phi_{S} \\
& +\left(-\alpha u_{w} d y_{C} L_{W W_{-} w}+\Gamma d y_{C} D_{W W_{-} w}\right) \phi_{W W} \\
& +\left(\alpha d y_{C}\left(u_{e} L_{W_{-} e}-u_{w} L_{W_{-} w}\right)+\Gamma d y_{C}\left(D_{W_{-} w}-D_{W_{-} e}\right)\right) \phi_{W} \\
& +\left(\begin{array}{l}
\alpha d y_{C}\left(u_{e} L_{C_{-} e}-u_{w} L_{C_{-} w}\right)+\alpha d x_{C}\left(v_{n} L_{C_{-} n}-v_{s} L_{C_{-} s}\right) \\
+\Gamma d y_{C}\left(D_{C_{-} w}-D_{C_{-} e}\right)+\Gamma d x_{C}\left(D_{C_{-} s}-D_{C_{-} n}\right) \\
+\alpha d x_{C} d y_{C} D_{4}
\end{array}\right) \phi_{C} \\
& +\left(\alpha d y_{C}\left(u_{e} L_{E_{-} e}-u_{w} L_{E_{-} w}\right)+\Gamma d y_{C}\left(D_{E_{-} w}-D_{E_{-} e}\right)\right) \phi_{E} \\
& +\left(\alpha u_{e} d y_{C} L_{E E_{-} e}-\Gamma d y_{C} D_{E E_{-} e}\right) \phi_{E E} \\
& +\left(\alpha d x_{C}\left(v_{n} L_{N_{-} n}-v_{s} L_{N_{-} s}\right)+\Gamma d x_{C}\left(D_{N_{-} s}-D_{N_{-} n}\right)\right) \phi_{N} \\
& +\left(\alpha v_{n} d x_{C} L_{N N_{-} n}-\Gamma d x_{C} D_{N N_{-} n}\right) \phi_{N N} \\
& =S_{\phi} d x_{C} d y_{C}-\alpha\left(D_{1} \cdot \phi_{C, 1}+D_{2} \cdot \phi_{C, 2}+D_{3} \cdot \phi_{C, 3}\right) d x_{C} d y_{C}
\end{aligned}
$$

The Eq. (21) can be written in the short form as

where

$$
a_{S S} \phi_{S S}+a_{S} \phi_{S}+a_{W W} \phi_{W W}+a_{W} \phi_{W}+a_{C} \phi_{C}+a_{E} \phi_{E}+a_{E E} \phi_{E E}+a_{N} \phi_{N}+a_{N N} \phi_{N N}=B
$$

$$
\begin{aligned}
a_{S S}= & -\alpha v_{s} d x_{C} L_{S S_{-} s}+\Gamma d x_{C} D_{S S_{-} s} \\
a_{S}= & \alpha d x_{C}\left(v_{n} L_{S_{-} n}-v_{s} L_{S_{-} s}\right)+\Gamma d x_{C}\left(D_{S_{-} s}-D_{S_{-} n}\right) \\
a_{W W}= & -\alpha u_{w} d y_{C} L_{W W_{-} w}+\Gamma d y_{C} D_{W W_{-} w} \\
a_{W}= & \alpha d y_{C}\left(u_{e} L_{W_{-} e}-u_{w} L_{W_{-} w}\right)+\Gamma d y_{C}\left(D_{W_{-} w}-D_{W_{-} e}\right) \\
a_{C}= & \alpha d y_{C}\left(u_{e} L_{C_{-} e}-u_{w} L_{C_{-} w}\right)+\alpha d x_{C}\left(v_{n} L_{C_{-} n}-v_{s} L_{C_{-} s}\right) \\
& +\Gamma d y_{C}\left(D_{C_{-} w}-D_{C_{-} e}\right)+\Gamma d x_{C}\left(D_{C_{-} s}-D_{C_{-} n}\right) \\
& +\alpha d x_{C} d y_{C} D_{4} \\
a_{E}= & \alpha d y_{C}\left(u_{e} L_{E_{-} e}-u_{w} L_{E_{-} w}\right)+\Gamma d y_{C}\left(D_{E_{-} w}-D_{E_{-} e}\right)
\end{aligned}
$$




$$
\begin{aligned}
& a_{E E}=\alpha u_{e} d y_{C} L_{E E_{-} e}-\Gamma d y_{C} D_{E E_{-} e} \\
& a_{N}=\alpha d x_{C}\left(v_{n} L_{N_{-} n}-v_{s} L_{N_{-} s}\right)+\Gamma d x_{C}\left(D_{N_{-} s}-D_{N_{-} n}\right) \\
& a_{N N}=\alpha v_{n} d x_{C} L_{N N_{-} n}-\Gamma d x_{C} D_{N_{-} n} \\
& B=\left(S_{\phi}-\alpha\left(D_{1} \cdot \phi_{C, 1}+D_{2} \cdot \phi_{C, 2}+D_{3} \cdot \phi_{C, 3}\right)\right) d x_{C} d y_{C}
\end{aligned}
$$

\subsection{Air Density Equation for the Body Force Term}

All air properties are the constant values except the density in the body force term in the momentum equation in the vertical direction. The constant values of the air properties are obtained from the National Institute of Standards and Technology (NIST) Standard Reference Database 23, Version 9.0 at the average temperature between the hot wall and the cold wall of the cavity, while the density in the body force term is a function of the temperature and pressure of fluid flow, which is also obtained from NIST Standard Reference Database 23, Version 9.0 by the two variables polynomial regression. The density equation for the body force term is formed as

$$
\rho=\sum_{j=1}^{n}\left(\left(\sum_{i=1}^{m}\left(a_{i j} T^{i-1}\right)\right) P^{j-1}\right)
$$

where $a_{i, j}$ is the polynomial regression coefficient. The coefficient of determination $\left(R^{2}\right)$ of the air density equation received from the two variables polynomial regression equals to 0.999 when $m$ and $n$ equal to 6 .

\section{Application Statement}

The application for the verification of the new scheme is two dimensional laminar natural convection in a square cavity. The width and height of the square cavity are designated as $b$. The vertical walls of the cavity are the differentially heated walls, while the horizontal walls are the adiabatic walls $\left(\frac{\partial T}{\partial y}=0\right)$. And, the left side wall of the cavity is the hot wall $\left(T=T_{H}\right)$, while the right wall of the cavity is the cold wall $\left(T=T_{C}\right)$. The no-slip condition $(u=v=0)$ is applied on any of the cavity walls as shown in Fig. 4.

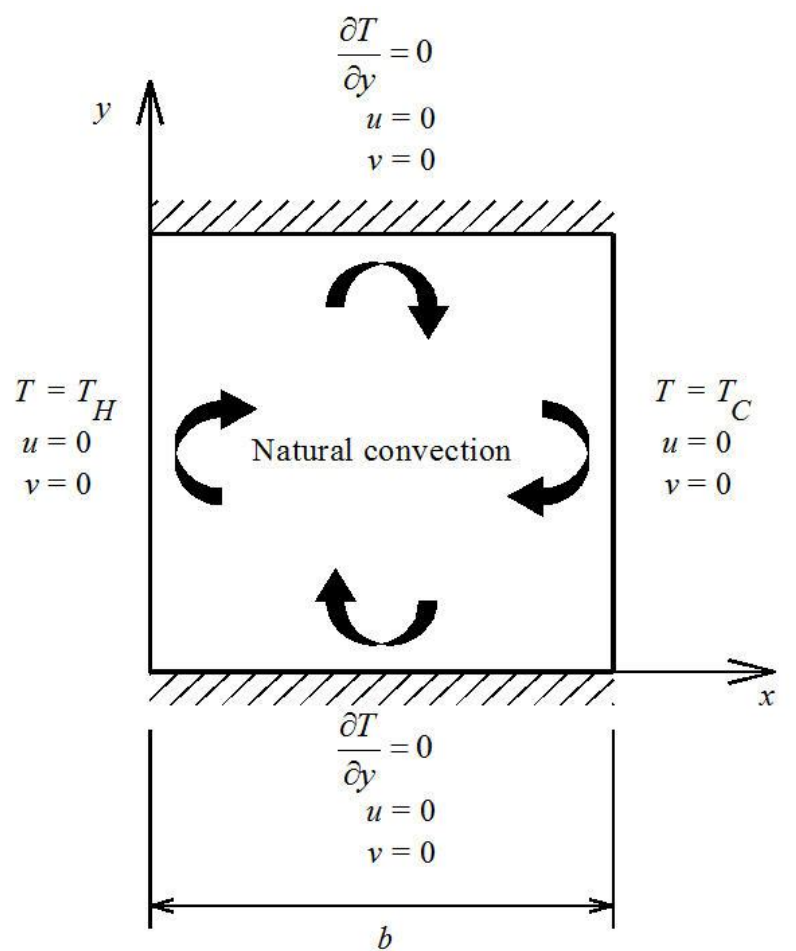

Fig. 4. Geometry of the application. 


\section{Numerical Method}

The finite volume method with the new scheme are employed to solve the partial differential equations of fluid flow. The SIMPLE algorithm is adopted to couple the continuity equation and the momentum equations to determine the pressure field of fluid flow. The transient condition approach to the steady-state condition is a strategy used to obtain the solutions in the steady-state condition. The relative residual values of the flow variables between the present iteration and the previous iteration are used as a convergent criterion. The convergent criterion is imposed to be less than or equal to $10^{-4}$. The convergent criterion is employed to check the convergence of the guess values and the computational values, and the convergence of the transient condition approach to steady-state condition. The Gauss's elimination method is employed to solve the system equations of the discretized equation of fluid flow.

\section{New Scheme Verification}

To ensure that the new scheme gives the correct solutions, the verification of the new scheme must be implemented. An in-house code conformed to the new scheme is developed from the FORTRAN programming language to achieve this purpose. The dimensionless parameter, Nusselt number $(\mathrm{Nu})$, is a solution obtained from the developed code which is compared to the benchmark numerical solutions and the published numerical solutions. For the transient condition approach to the steady-state condition, the time-step of the code is $0.01 \mathrm{~s}$.

\subsection{Heat Transfer}

The average Nusselt number of the cavity is given by

$$
\overline{N u}=\frac{1}{b} \int_{0}^{b} \frac{\left(\left.q^{\prime \prime}\right|_{x=0}+\left.q^{\prime \prime}\right|_{x=b}\right)}{2 q_{r e f}^{\prime \prime}} d y
$$

The heat flux on the vertical wall of the cavity is calculated by

$$
q^{\prime \prime}=-k_{A} \frac{d T}{d x}
$$

where $k_{A}$ is the thermal conductivity of air in the cavity. The reference heat flux is expressed as

$$
q_{r e f}^{\prime \prime}=k_{A} \frac{\left(T_{H}-T_{C}\right)}{b}
$$

\subsection{Grid Independence Test}

The grid independence tests are performed to ensure that there are not any variations of solutions because of grid sizes. The temperature gradients near the cavity walls are steep, so the non-uniform grids are employed. The fine grids near the cavity walls and the coarse grids in the core area of the cavity are used with the ratio of the distance between the coarse grids to the fine grids is 1.2. Since the average Nusselt number is a function of the Rayleigh number $(R a)$, the tests are implemented with the three values of the Rayleigh number. The percentage change of the average Nusselt number values between the sequential test of the grid sizes is a criterion for the tests.

From Table 1, The average Nusselt numbers change less than $0.100 \%$ when the grid sizes are $32 \times 32$, $37 \times 37$ and $42 \times 42$ for the Rayleigh numbers equal to $10^{4}, 10^{5}$ and $10^{6}$, respectively. So, the grid sizes $32 \times 32$, $37 \times 37$ and $42 \times 42$ are used for verification for the Rayleigh numbers equal to $10^{3}-10^{4}, 2 \times 10^{4}-10^{5}$ and $2 \times 10^{5}$ $10^{6}$, respectively. 
Table 1. Grid independence tests for the application.

\begin{tabular}{cccc}
\hline $\boldsymbol{R} \boldsymbol{a}$ & Grid size & $\overline{\mathrm{Nu}}$ & Change (\%) \\
\hline \multirow{3}{*}{$10^{4}$} & $12 \times 12$ & 2.236 & - \\
& $17 \times 17$ & 2.249 & 0.578 \\
& $22 \times 22$ & 2.259 & 0.399 \\
& $27 \times 27$ & 2.261 & 0.133 \\
& $32 \times 32$ & 2.263 & 0.088 \\
\hline \multirow{2}{*}{$10^{5}$} & $22 \times 22$ & 4.493 & - \\
& $27 \times 27$ & 4.513 & 0.443 \\
& $32 \times 32$ & 4.521 & 0.177 \\
\multirow{2}{*}{$10^{6}$} & $37 \times 37$ & 4.523 & 0.044 \\
& $32 \times 32$ & 8.830 & - \\
& $37 \times 37$ & 8.849 & 0.213 \\
\hline
\end{tabular}

\subsection{Results and Discussion}

The comparison of the solutions of the new scheme to the benchmark numerical solutions and the published numerical solutions was performed as shown in Table 2 . The maximum difference between the solutions of the new scheme and the benchmark numerical solutions is 1.619 percent, while the maximum difference between the solutions of the new scheme and the published numerical solutions is 6.922 percent. But most of the percentage differences are very small, so these results demonstrate that the new scheme is valid.

Figures 5-9 show the contours of the velocity components in the horizontal direction and the vertical directions, the temperature, the stream function and the vorticity of fluid flow in the square cavity at the different Rayleigh number values. The figures demonstrate that the solutions from the new scheme agree well with the solutions of De Vahl Davis [15].

The variation of the dimensionless temperature $\left(T^{* *}=\frac{T-T_{C}}{T_{H}-T_{C}}\right)$ in the horizontal direction in the square cavity on the bottom wall $\left(T^{* *} B\right)$, the mid-plane $\left(T^{* *} M\right)$ and the top wall $\left(T^{* *} T\right)$ of the cavity for the different Rayleigh number values is shown in Fig. 10. The gradient of the dimensionless temperature near the vertical wall of the cavity increases, when the Rayleigh number value increases. In addition, Fig. 11 and Fig. 12 present the variation of the dimensionless velocity components $\left(u^{* *}=\frac{u}{\alpha / b}\right.$ and $\left.v^{* *}=\frac{v}{\alpha / b}\right)$ in the horizontal and vertical direction on the middle plane of the cavity in the vertical and horizontal directions, respectively. The velocities near the walls of the cavity also increase, when the Rayleigh number value increases. The velocity at the center of the cavity is zero. Fig. 10, Fig. 11 and Fig. 12 tend according to the earlier literature $[16,18,19]$.

A new correlation between the Nusselt number and the Rayleigh number from the solutions conforming to the new scheme is established in a power-law form. The number of data collected to perform curve fitting to determine the coefficients of the correlation is 28 sets. The coefficient of determination $\left(R^{2}\right)$ of the new correlation is 0.999 . The comparison of the coefficients of the correlations between the present work and the other works $[17,18]$ is shown in Table 3 . The differences of the coefficients of the correlations are less than or equal to 4.380 percent. Therefore, it gives credence to the new scheme. 
Table 2. Comparison of the solutions of the new scheme to the benchmark numerical solutions and the published numerical solutions.

\begin{tabular}{|c|c|c|c|c|c|c|c|c|}
\hline & \multicolumn{2}{|c|}{$R a=10^{3}$} & \multicolumn{2}{|c|}{$R a=10^{4}$} & \multicolumn{2}{|c|}{$R a=10^{5}$} & \multicolumn{2}{|c|}{$R a=10^{6}$} \\
\hline & $\overline{N u}$ & $\begin{array}{c}\text { Difference } \\
(\%)\end{array}$ & $\overline{N u}$ & $\begin{array}{c}\text { Difference } \\
(\%)\end{array}$ & $\overline{\mathrm{Nu}}$ & $\begin{array}{c}\text { Difference } \\
(\%)\end{array}$ & $\overline{N u}$ & $\begin{array}{c}\text { Difference } \\
(\%)\end{array}$ \\
\hline $\begin{array}{c}\text { Present } \\
\text { work }\end{array}$ & 1.118 & - & 2.263 & - & 4.523 & - & 8.856 & - \\
\hline [15] & 1.118 & 0.000 & 2.234 & 0.884 & 4.519 & 0.088 & 8.800 & 0.632 \\
\hline [16] & - & - & 2.2415 & 0.950 & - & - & 8.7126 & 1.619 \\
\hline [17] & 1.108 & 0.894 & 2.201 & 2.740 & 4.430 & 2.056 & 8.754 & 1.152 \\
\hline [18] & 1.114 & 0.358 & 2.245 & 0.795 & 4.510 & 0.287 & 8.806 & 0.565 \\
\hline [19] & 1.121 & 0.268 & 2.286 & 1.016 & 4.546 & 0.515 & 8.652 & 2.304 \\
\hline$[20]$ & 1.112 & 0.537 & 2.168 & 4.198 & 4.228 & 6.522 & 8.243 & 6.922 \\
\hline
\end{tabular}

Table 3. Comparison of the coefficients of the power-law correlation: $\overline{N u}=a(R a)^{b}$.

\begin{tabular}{cccc}
\hline & $a$ & $b$ & Range \\
\hline Present work & 0.137 & 0.303 & $10^{3} \leq R a \leq 10^{6}$ \\
{$[17]$} & 0.143 & 0.299 & $10^{3} \leq R a \leq 10^{6}$ \\
{$[18]$} & 0.142 & 0.299 & $10^{3} \leq R a \leq 10^{6}$ \\
\hline
\end{tabular}

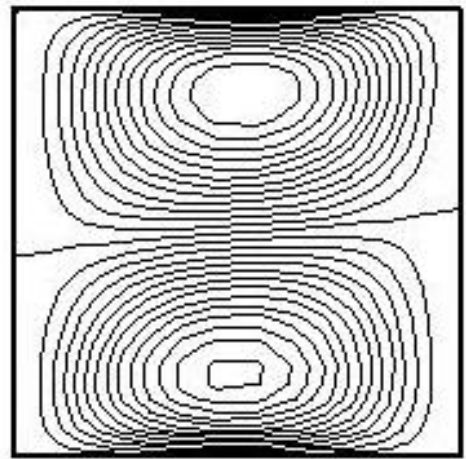

(a)

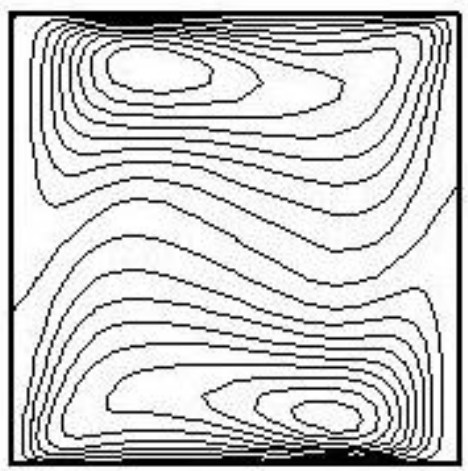

(c)

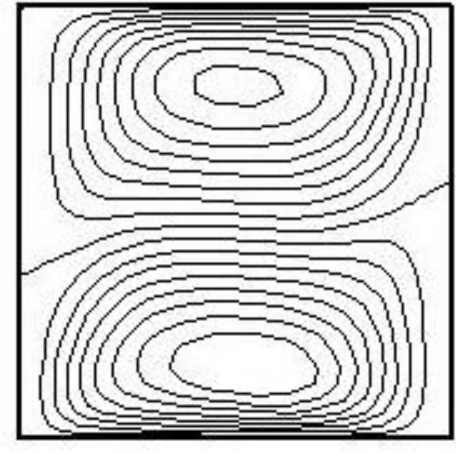

(b)

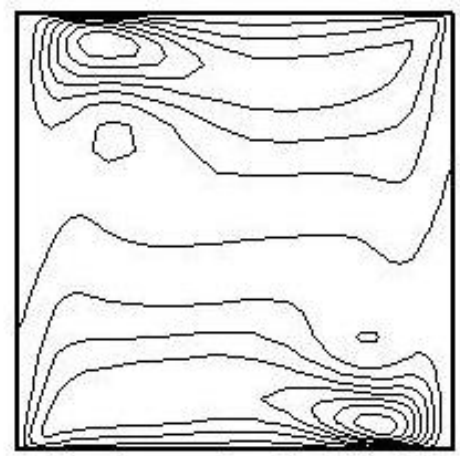

(d)

Fig. 5. Contours of the velocity component in the horizontal direction of fluid flow in the square cavity for: (a) $R a=10^{3}$, (b) $R a=10^{4}$, (c) $R a=10^{5}$, (d) $R a=10^{6}$. 


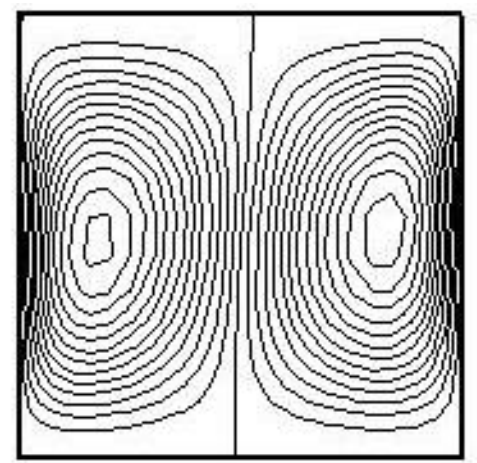

(a)

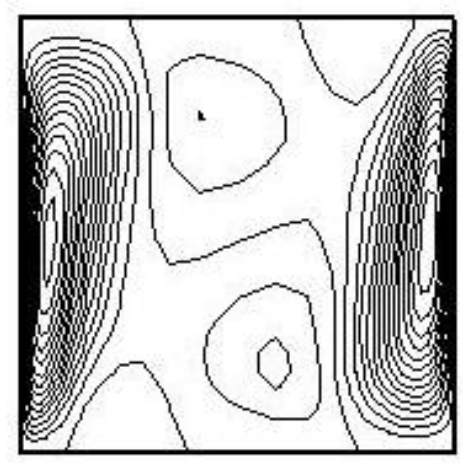

(c)

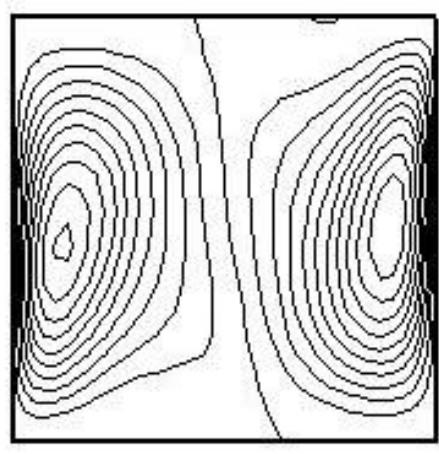

(b)

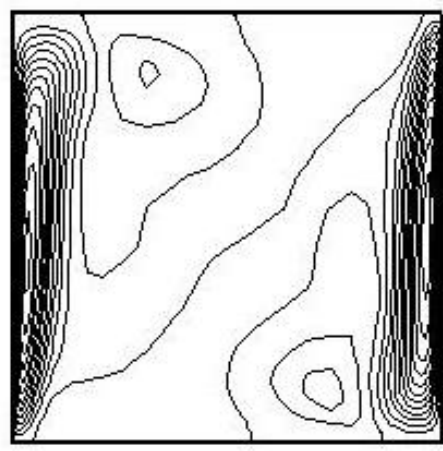

(d)

Fig. 6. Contours of the velocity component in the vertical direction of fluid flow in the square cavity for: (a) $R a=10^{3}$, (b) $R a=10^{4}$, (c) $R a=10^{5}$, (d) $R a=10^{6}$.

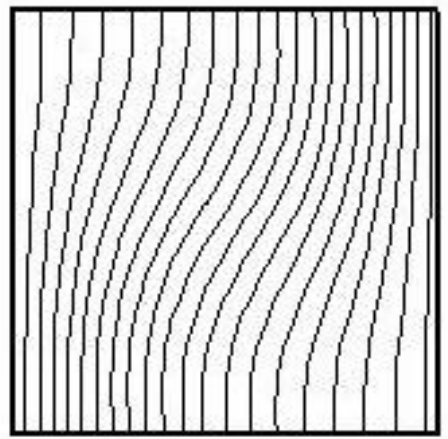

(a)

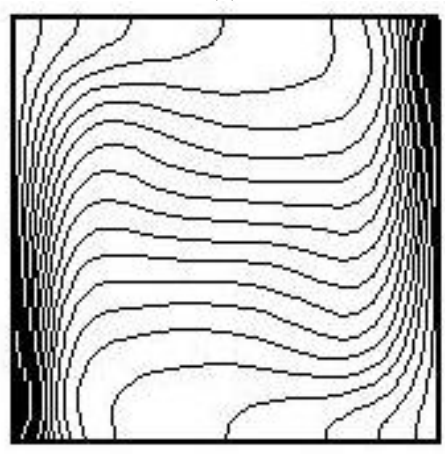

(c)

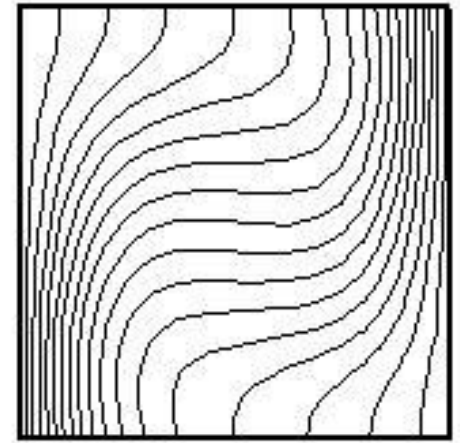

(b)

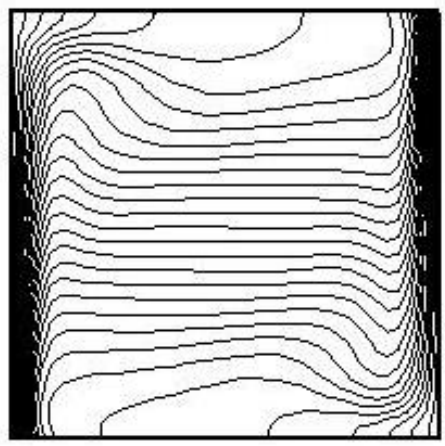

(d)

Fig. 7. Contours of the temperature of fluid flow in the square cavity for: (a) $R a=10^{3}$, (b) $R a=10^{4}$, (c) $R a=10^{5}$, (d) $R a=10^{6}$. 


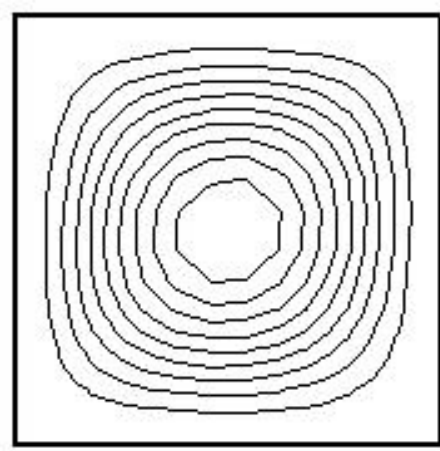

(a)

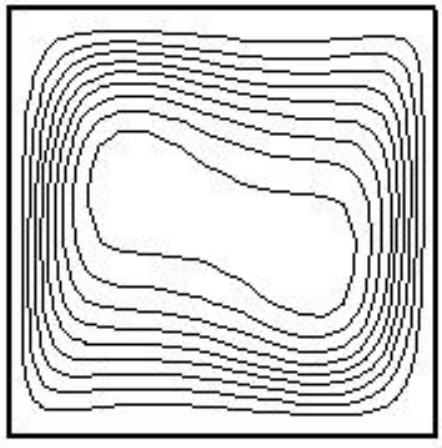

(c)

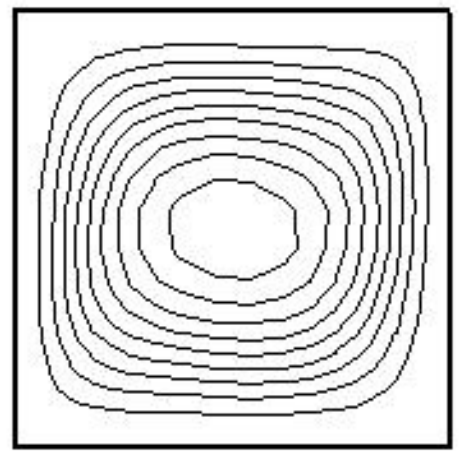

(b)

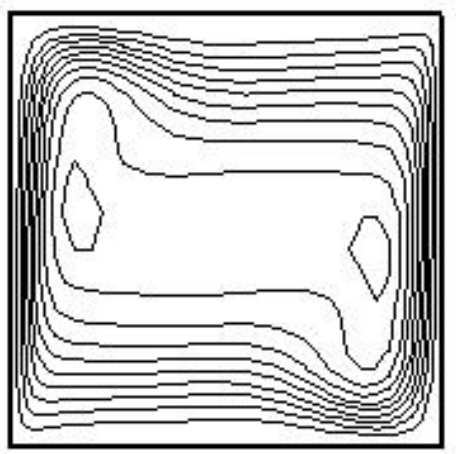

(d)

Fig. 8. Contours of the stream function of fluid flow in the square cavity for: (a) $R a=10^{3}$, (b) $R a=$ $10^{4}$, (c) $R a=10^{5}$, (d) $R a=10^{6}$.

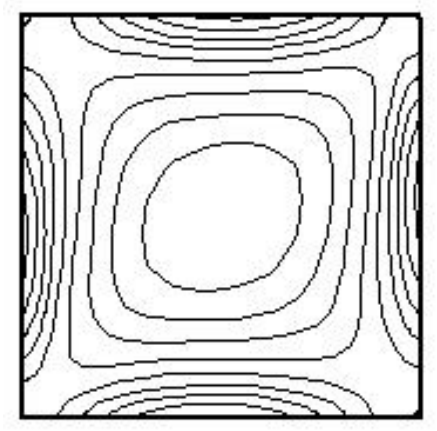

(a)

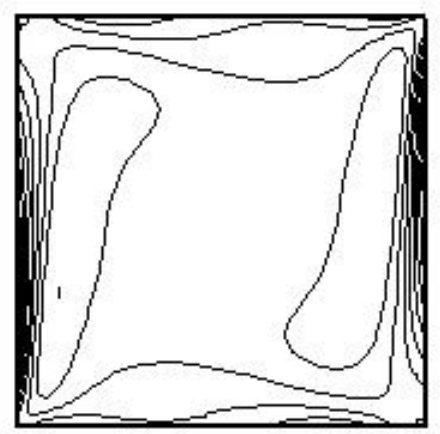

(c)

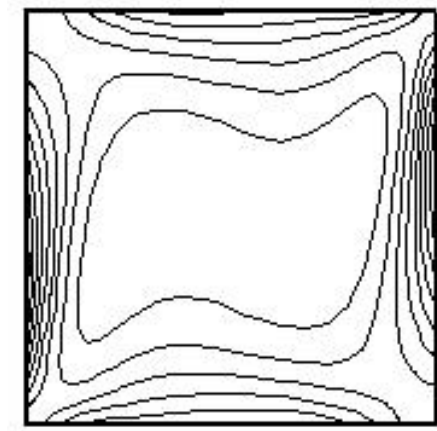

(b)

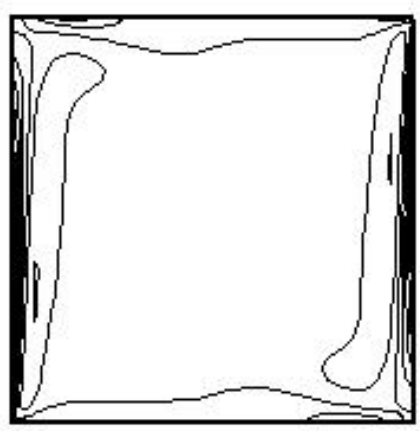

(d)

Fig. 9. Contours of the vorticity of fluid flow in the square cavity for: (a) $R a=10^{3}$, (b) $R a=10^{4}$, (c) $R a=10^{5}$, (d) $R a=10^{6}$. 


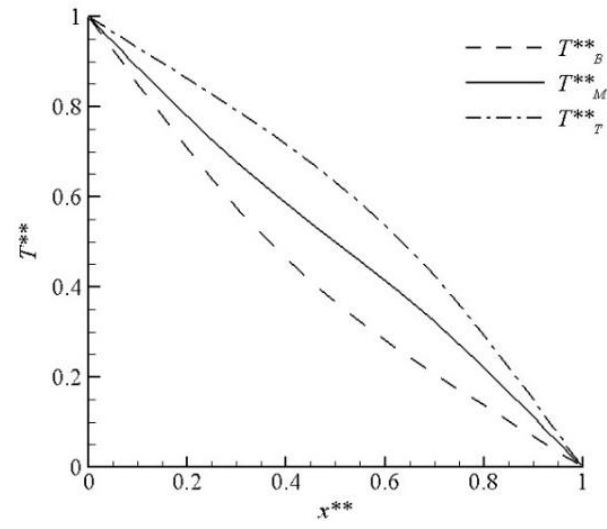

(a)

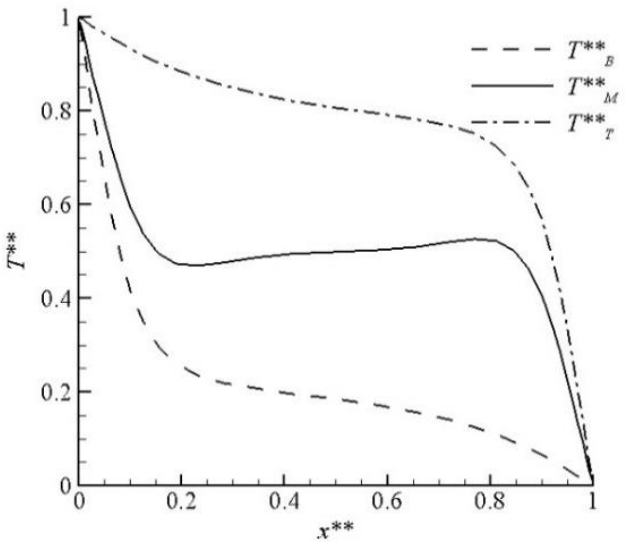

(c)

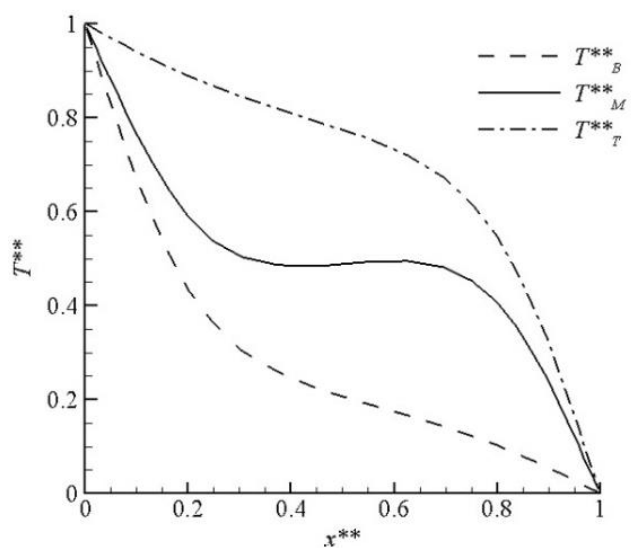

(b)

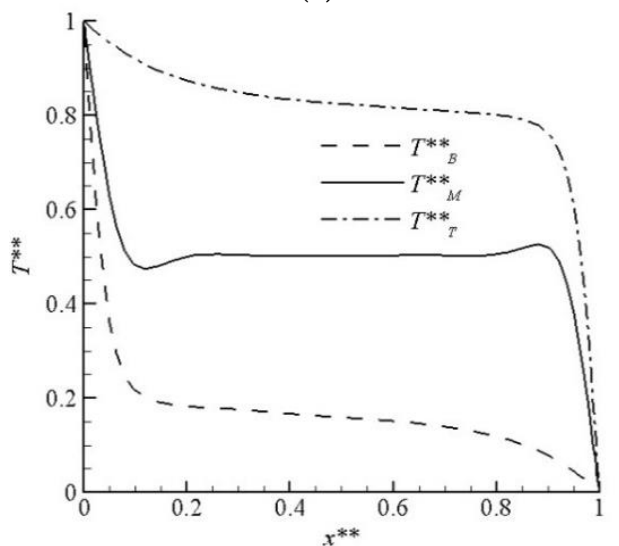

(d)

Fig. 10. Variation of the dimensionless temperature on the bottom wall, mid-plane and top wall of the square cavity for: (a) $R a=10^{3}$, (b) $R a=10^{4}$, (c) $R a=10^{5}$, (d) $R a=10^{6}$.

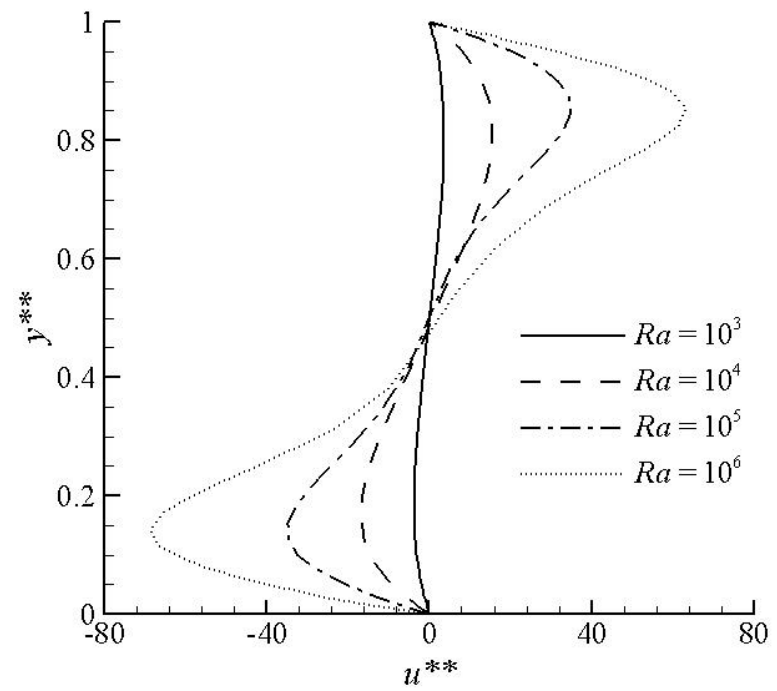

Fig. 11. Variation of the dimensionless velocity component in the horizontal direction on the middle plane of the cavity in the vertical direction. 


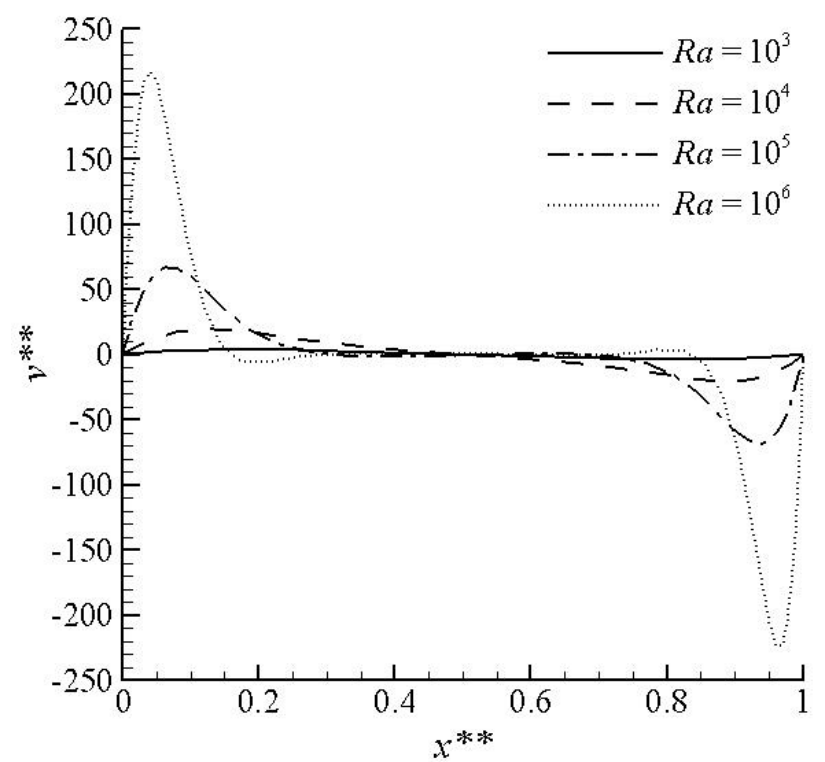

Fig. 12. Variation of the dimensionless velocity component in the vertical direction on the middle plane of the cavity in the horizontal direction.

\section{Conclusion}

The new scheme is proposed and verified with an application, two dimensional natural convection in a square cavity. The Lagrange interpolating polynomial with a setting of zero for the spatial domain at the cell faces, and the present time at the cell center is adopted to estimate the values and the derivative values of the variables for the new scheme. The robustness of the new scheme is, it is easier to determine the coefficients of the scheme for high order accuracy with non-uniform grids than with existing schemes and can be extended to be the $n$ order accuracy. The verification of the new scheme was performed by comparing the solutions obtained from the new scheme to the benchmark numerical solutions and the published numerical solutions based on the application. The results of the comparison in many aspects of the solutions give credence to the new scheme and show that it is valid. In addition, the new correlation between the Nusselt number obtained from the new scheme and the Rayleigh number is established by using the new scheme to simulate the application. The new correlation can be written as $\overline{N u}=0.137 R a^{0.303}$, $10^{3} \leq R a \leq 10^{6}$.

\section{Acknowledgement}

The authors wish to acknowledge Thai National e-Science Infrastructure Consortium for providing computing resources that have contributed to the research results reported within this paper.

\section{References}

[1] D. Gaitonde and J. S. Shang, "Optimized compact-difference-based finite volume schemes for linear wave phenomena," J. Comput. Phys., vol. 138, pp. 617-643, 1997.

[2] J. M. C. Pereira, M. H. Kobayashi, and J. C. F. Pereira, "A fourth-order-accurate finite volume compact method for the incompressible Navier-Stokes solutions," J. Comput. Phys., vol. 167, pp. 217 243, 2001.

[3] M. Piller and E. Stalio, "Finite-volume compact schemes on staggered grids," J. Comput. Phys., vol. 197, pp. 299-340, 2004.

[4] N. A. Kampanis and J. A. Ekaterinaris, "A staggered grid, high-order accurate method for the incompressible Navier-Stokes equations,” J. Comput. Phys., vol. 215, pp. 589-613, 2006.

[5] Y. Guo, T. Xiong, and Y. Shi, "A positivity-preserving high order finite volume compact-WENO scheme for compressible Euler equations," J. Comput. Phys., vol. 274, pp. 505-523, 2014. 
[6] X. Zeng, "A high-order hybrid finite difference-finite volume approach with application to inviscid compressible flow problems: A preliminary study," Comput. Fluids, vol. 98, pp. 91-110, 2014.

[7] R. K. Shukla and X. Zhong, "Derivation of high-order compact finite difference schemes for nonuniform grid using polynomial interpolation," J. Comput. Phys., vol. 204, pp. 404-429, 2005.

[8] R. K. Shukla, M. Tatineni, and X. Zhong, "Very high-order compact finite difference schemes on non-uniform grids for incompressible Navier-Stokes equations," J. Comput. Phys., vol. 224, pp. 1064 1094, 2007.

[9] S. Ii and F. Xiao, "High order multi-moment constrained finite volume methed. Part I: Basic formulation," J. Comput. Phys., vol. 228, pp. 3669-3707, 2009.

[10] A. Hokpunna and M. Manhart, "Compact fourth-order finite volume method for numerical solutions of Navier-Stokes equations on staggered grids," J. Comput. Phys., vol. 229, pp. 7545-7570, 2010.

[11] A. Beljadid, A. Mohammadian, and H. M. Qiblawey, "An unstructured finite volume method for large-scale shallow flows using the fourth-order Adame scheme," Comput. Fluids, vol. 88, pp. 579-589, 2013.

[12] W. Chen, J. C. Chen, and E. Y. Lo, "An interpolation based finite difference method on non-uniform grid for solving Navier-Stokes equations," Comput. Fluids, vol. 101, pp. 273-290, 2014.

[13] L. Ramirez, X. Nogueira, S. Khelladi, J.-C. Chassaing, and I. Colominas, "A new high-order finite volume method based on Moving Least Square for the resolution of incompressible Navier-Stokes equations on unstructured grids," Comput. Methods appl. Mech. Eng., vol. 278, pp. 883-901, 2014.

[14] A. Bairi and E. Zarco-Pernia, "A review on natural convection in enclosure for engineering applications. The particular case of the parallelogrammic diode cavity," Appl. Therm. Eng., vol. 63, pp. 304-322, 2014.

[15] G. D. V. Davis, "Natural convection of air in a square cavity a bench mark numerical solution," Int. J. Numer. Methods Fluids, vol. 3, pp. 249-264, 1983.

[16] T. Saitoh and K. Hirose, "High-accuracy bench mark solutions to natural convection in a square cavity," Comput. Mech., vol. 4, pp. 417-427, 1989.

[17] N. C. Markatos and K. A. Pericleous, "Laminar and turbulent natural convection in an enclosed cavity.," Int. J. Heat Mass Transf., vol. 27, pp. 755-772, 1984.

[18] G. Barakos, E. Mitsoulis, and D. Assimacopoulos, "Natural convection flow in a square cavity revised: Laminar and turbulent models with wall functions," Int. J. Numer. Methods Fluids, vol. 18, pp. 695-719, 1994.

[19] H. N. Dixit and V. Babu, "Simulation of high Rayleigh number natural convection in a square cavity using the lattice Boltzmann method," Int. J. Heat Mass Transf., vol. 49, pp. 727-739, 2006.

[20] A. Bairi, "Nusselt-Rayleigh correlations for design of industrial elements: Experimental and numerical investigation of natural convection in tilted square air filled enclosures," Energy Convers. Management, vol. 49, pp. 771-782, 2008.

[21] S. C. Chapra and R. P. Canale, Numerical Methods for Engineers, 2nd ed. New York: McGraw-Hill, 1990.

[22] F. H. Harlow and J. E. Welch, "Numerical calculation of time-dependent viscous incompressible flow of fluid with free surface," Phys. Fluids, vol. 8, pp. 2182-2189, 1965.

[23] K. A. Hoffmann and S. T. Chiang, Computational fluid dynamics, 4th ed. U.S.A.: Engineering Education System, 2000. 
Appendix: Expression of the Order Accuracy for the Value Estimated by Using the Lagrange Interpolation Polynomial

From the Taylor series expansion and Fig. A-1, the variable values of the cell centers $\left(F_{W W}, F_{W}, F_{C}\right.$ and $\left.F_{E}\right)$ can be approximated from the variable value at cell face $\left(F_{w}\right)$ as shown below:

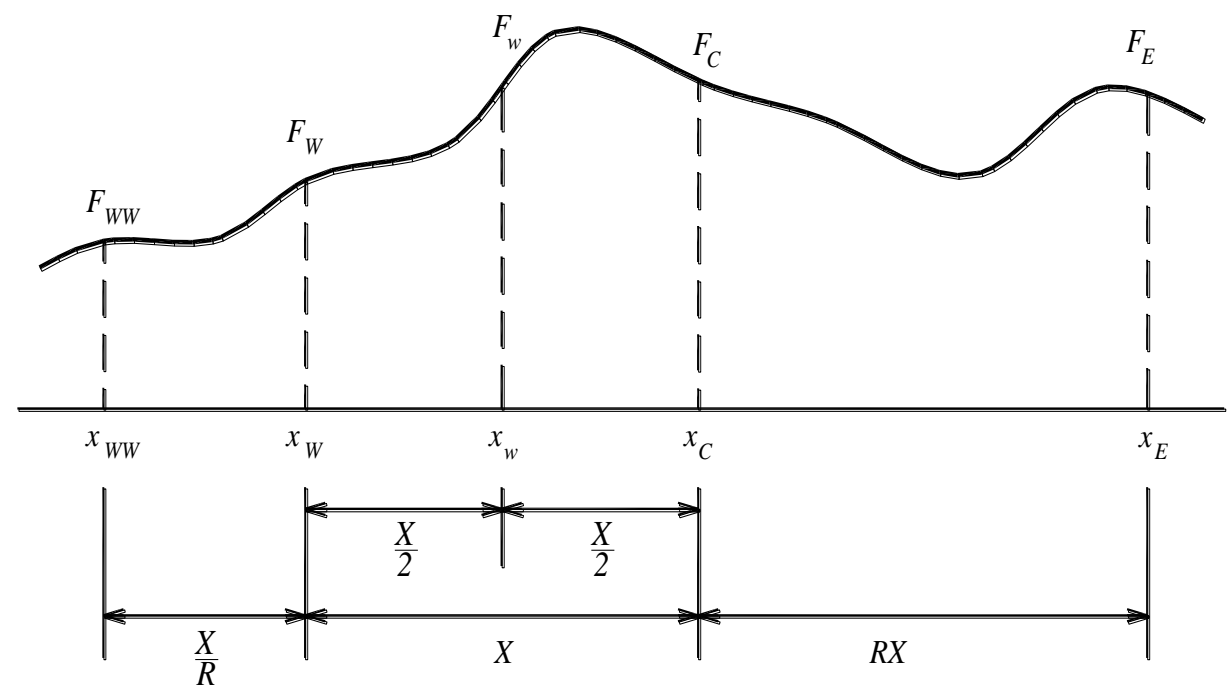

Fig. A-1. Value $F$ of variables at the western cell face on the spatial domain in the $x$ direction and the distance between the cell centers.

$$
\begin{gathered}
F_{W W}=F_{w}-\left(\frac{X}{R}+\frac{X}{2}\right) \cdot \frac{\partial F_{w}}{\partial x}+\frac{\left(\frac{X}{R}+\frac{X}{2}\right)^{2}}{2 !} \cdot \frac{\partial^{2} F_{w}}{\partial x^{2}} \\
-\frac{\left(\frac{X}{R}+\frac{X}{2}\right)^{3}}{3 !} \cdot \frac{\partial^{3} F_{w}}{\partial x^{3}}+\frac{\left(\frac{X}{R}+\frac{X}{2}\right)^{4}}{4 !} \cdot \frac{\partial^{4} F_{w}}{\partial x^{4}}+\ldots \\
F_{W}=F_{w}-\left(\frac{X}{2}\right) \cdot \frac{\partial F_{w}}{\partial x}+\frac{\left(\frac{X}{2}\right)^{2}}{2 !} \cdot \frac{\partial^{2} F_{w}}{\partial x^{2}}-\frac{\left(\frac{X}{2}\right)^{3}}{3 !} \cdot \frac{\partial^{3} F_{w}}{\partial x^{3}}+\frac{\left(\frac{X}{2}\right)^{4}}{4 !} \cdot \frac{\partial^{4} F_{w}}{\partial x^{4}}+\ldots \\
F_{C}=F_{w}+\left(\frac{X}{2}\right) \cdot \frac{\partial F_{w}}{\partial x}+\frac{\left(\frac{X}{2}\right)^{2}}{2 !} \cdot \frac{\partial^{2} F_{w}}{\partial x^{2}}+\frac{\left(\frac{X}{2}\right)^{3}}{3 !} \cdot \frac{\partial^{3} F_{w}}{\partial x^{3}}+\frac{\left(\frac{X}{2}\right)^{4}}{4 !} \cdot \frac{\partial^{4} F_{w}}{\partial x^{4}}+\ldots \\
F_{E}= \\
F_{w}+\left(\frac{X}{2}+R X\right) \cdot \frac{\partial F_{w}}{\partial x}+\frac{\left(\frac{X}{2}+R X\right)^{2}}{2 !} \cdot \frac{\partial^{2} F_{w}}{\partial x^{2}} \\
+\frac{\left(\frac{X}{2}+R X\right)^{3}}{3 !} \cdot \frac{\partial^{3} F_{w}}{\partial x^{3}}+\frac{\left(\frac{X}{2}+R X\right)^{4}}{4 !} \cdot \frac{\partial^{4} F_{w}}{\partial x^{4}}+\ldots
\end{gathered}
$$

Multiply Eq. (A-1a), Eq. (A-1b), Eq. (A-1c) and Eq. (A-1d) by $\frac{-R^{3}(1+2 R)}{8(1+R)\left(1+R+R^{2}\right)}, \frac{(2+R)(1+2 R)}{8(1+R)}$, $\frac{(2+R)(1+2 R)}{8 R(1+R)}$ and $\frac{-(2+R)}{8 R(1+R)\left(1+R+R^{2}\right)}$, respectively and sum them together, so that the result is 


$$
\begin{aligned}
F_{w}= & \frac{-R^{3}(1+2 R)}{8(1+R)\left(1+R+R^{2}\right)} \cdot F_{W W}+\frac{(2+R)(1+2 R)}{8(1+R)} \cdot F_{W}+\frac{(2+R)(1+2 R)}{8 R(1+R)} \cdot F_{C} \\
& +\frac{-(2+R)}{8 R(1+R)\left(1+R+R^{2}\right)} \cdot F_{E}+E\left(x^{4}\right)+\ldots
\end{aligned}
$$

where $R$ is the ratio of the distance between the coarse grids to the fine grids and $E\left(x^{4}\right)$ is the truncation error of the variable value approximated by using the Tayor series expansion.

From the Lagrange interpolation polynomial with a setting of zero for the spatial domain at the cell face $\left(x_{w}=0\right)$ and Fig. A-1, The variable value at the cell face can be estimated from the variable values of the neighbor cells.

$$
\begin{aligned}
F_{w}= & -\frac{x_{W} \cdot x_{C} \cdot x_{E}}{\left(x_{W W}-x_{W}\right)\left(x_{W W}-x_{C}\right)\left(x_{W W}-x_{E}\right)} F_{W W} \\
& -\frac{x_{W W} \cdot x_{C} \cdot x_{E}}{\left(x_{W}-x_{W W}\right)\left(x_{W}-x_{C}\right)\left(x_{W}-x_{E}\right)} F_{W} \\
& -\frac{x_{W W} \cdot x_{W} \cdot x_{E}}{\left(x_{C}-x_{W W}\right)\left(x_{C}-x_{W}\right)\left(x_{C}-x_{E}\right)} F_{C} \\
& -\frac{x_{W W} \cdot x_{W} \cdot x_{C}}{\left(x_{E}-x_{W W}\right)\left(x_{E}-x_{W}\right)\left(x_{E}-x_{C}\right)} F_{E}
\end{aligned}
$$

Substitute the values of $x_{W W}, x_{W}, x_{C}$ and $x_{E}$ by $-\left(\frac{X}{R}+\frac{X}{2}\right),-\left(\frac{X}{2}\right),\left(\frac{X}{2}\right)$ and $\left(\frac{X}{2}+R X\right)$, respectively into Eq. (A-3), so that the result becomes

$$
\begin{aligned}
F_{w}= & \frac{-R^{3}(1+2 R)}{8(1+R)\left(1+R+R^{2}\right)} \cdot F_{W W}+\frac{(2+R)(1+2 R)}{8(1+R)} \cdot F_{W} \\
& +\frac{(2+R)(1+2 R)}{8 R(1+R)} \cdot F_{C}+\frac{-(2+R)}{8 R(1+R)\left(1+R+R^{2}\right)} \cdot F_{E}
\end{aligned}
$$

The error of the variable value estimated by using the Lagrange interpolation polynomial is

$$
\operatorname{ERROR}_{L}=\left(F_{w}\right)_{T}-\left(F_{w}\right)_{L}
$$

where $\left(F_{w}\right)_{T}$ and $\left(F_{w}\right)_{L}$ are the values estimated by using the Taylor series expansion and the Lagrange interpolation polynomial, respectively. Substitute the values of $\left(F_{w}\right)_{T}$ and $\left(F_{w}\right)_{L}$ from Eq. (A-2) and Eq. (A-4), respectively into Eq. (A-5) so that it yields

$$
\text { ERROR }_{L}=E\left(x^{4}\right)
$$

From Eq. (A-6), the accuracy is the fourth-order for the variable value estimated from the four variable values of the neighbor cells by using the Lagrange interpolation polynomial. 